\title{
Microstructure Evolution of Discontinuous Precipitation and Coarsening of Co Rod-like Precipitates in Supersaturated Cu-Co alloys
}

\author{
${ }^{1}$ N. M. Suguihiro, ${ }^{2}$ I. G. Solórzano, ${ }^{1}$ E. B. Saitvitch \\ ${ }^{1}$ Centro Brasileiro de Pesquisas Físicas, Rio de Janeiro, Brazil \\ ${ }^{2}$ Department of Chemical and Materials Engineering - PUC-Rio de Janeiro, Brazil
}

Discontinuous precipitation (DP) is a solid state reaction in which precipitation takes place in a moving interface, generally a moving, incoherent, grain boundary. The transformation product is arrays of precipitates growing cooperatively and perpendicular to the moving interface. Among hundreds of alloy systems in which DP takes place, Cu-Co alloy is of great importance due to ferromagnetic character of Co precipitates. In this alloy system, anisotropic arrangement of precipitates generates magnetic anisotropy and consequently drastic changes in its magnetic properties. It is known that in diluted $\mathrm{Cu}-\mathrm{Co}$ alloys, DP takes place at relatively low temperatures, ranging from 450 to $700^{\circ} \mathrm{C}$ with Co rod-like precipitates coherent with the matrix [1]. However, interesting magnetic properties have been reported in supersaturated $\mathrm{Cu}-\mathrm{Co}$ alloys in condition in which DP is the dominant mode of precipitation after isothermal aging treatments [2, 3]. For this reason, it is of fundamental importance to understand the development of DP and coarsening in $\mathrm{Cu}-$ Co alloys.

In this paper we investigated the growth of DP and coarsening in supersaturated Cu-10at. \%Co alloys upon isothermal aging at temperatures ranging from 450 to $650^{\circ} \mathrm{C}$, for periods of $5,10,30$ and $60 \mathrm{~min}$. Due to nanometric size of precipitates (about 2nm in diameter), a TEM/STEM JEOL JEM $2100 \mathrm{~F}$ operating at $200 \mathrm{kV}$ under conventional, scanning and analytical transmission electron microscopy was used to analyze the microstructure in detail. Nano beam diffraction (NBD) mapping NANOMEGAS technique has been used to evaluate orientation relationships between precipitate colonies and parent matrix as well as preferential directions of discontinuous reactions upon grain boundary migration.

Results show that DP was the dominant mode of precipitation. Discontinuous precipitates are rodlike Co-rich, fully coherent with matrix with fcc structure, as confirmed by diffraction patterns. We verified a good stability of these precipitates at $450^{\circ} \mathrm{C}$ up to $60 \mathrm{~min}$ of aging, with DP development in all high angle grain boundaries. At higher temperatures, coarsening takes place rapidly consuming discontinuous precipitates. This coarsening is also grain boundary driven, so-called discontinuous coarsening (DC): the grain boundary moves consuming DP colonies replacing them by coarsen rodlike precipitates, with fcc structure and still coherent with matrix at early stages. However, under aging for longer periods of time DC precipitates lose coherency and change orientation in a similar fashion as demonstrated by Takeda for coherency loss of homogeneous Co precipitates [4]. Fig. 1a and b show well-defined DP and DC colonies respectively. Fig.2a shows the coherency loss of a rodlike DC precipitate by emission of dislocation loops, seen as the ring-like contrast. EDS Co mapping in Fig. 2b confirm the presence of precipitate phase $\beta$. After longer aging times, DC $\beta$ precipitates become incoherent with the matrix $\alpha$ and change orientation adopting the orientation relationship $(100)_{\alpha} / /(110)_{\beta}$, as can be seen in the NBD mapping in Fig.3.

\section{References}

[1] A. Perovic, G. R. Purdy, Acta Metall. 29, (1980) 53.

[2]E. F. Ferrari, W. C. Nunes, M. A. Novak, J Appl. Phys. 86 (1999) 3010.

[3] N. M. Suguihiro et al. J. Mat. Sci. 49 (2014) 6167.

[4] M. Takeda et al. Phys. Stat. Sol. 168 (1998) 27. 
[5] Authors acknowledge CNPq for financial support.
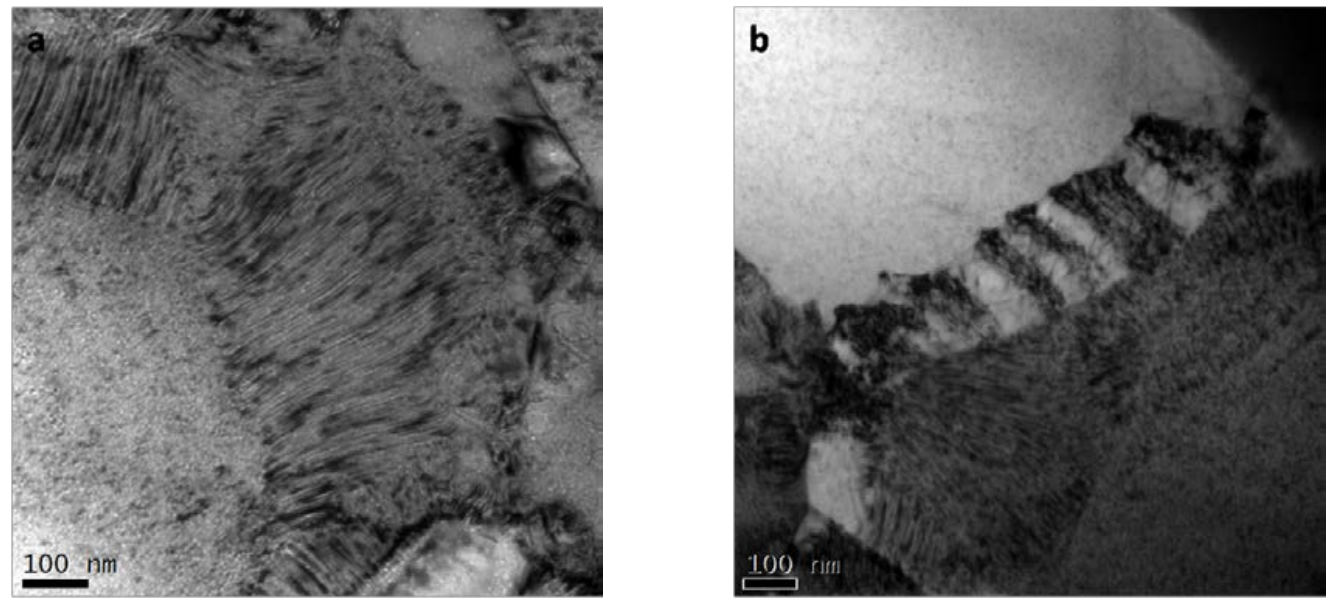

Figure 1 - TEM bright field images. (a) DP colony in sample aged at $550^{\circ} \mathrm{C}$ for $60 \mathrm{~min}$. (b) Discontinuous coarsening after $30 \mathrm{~min}$ at $550^{\circ} \mathrm{C}$.
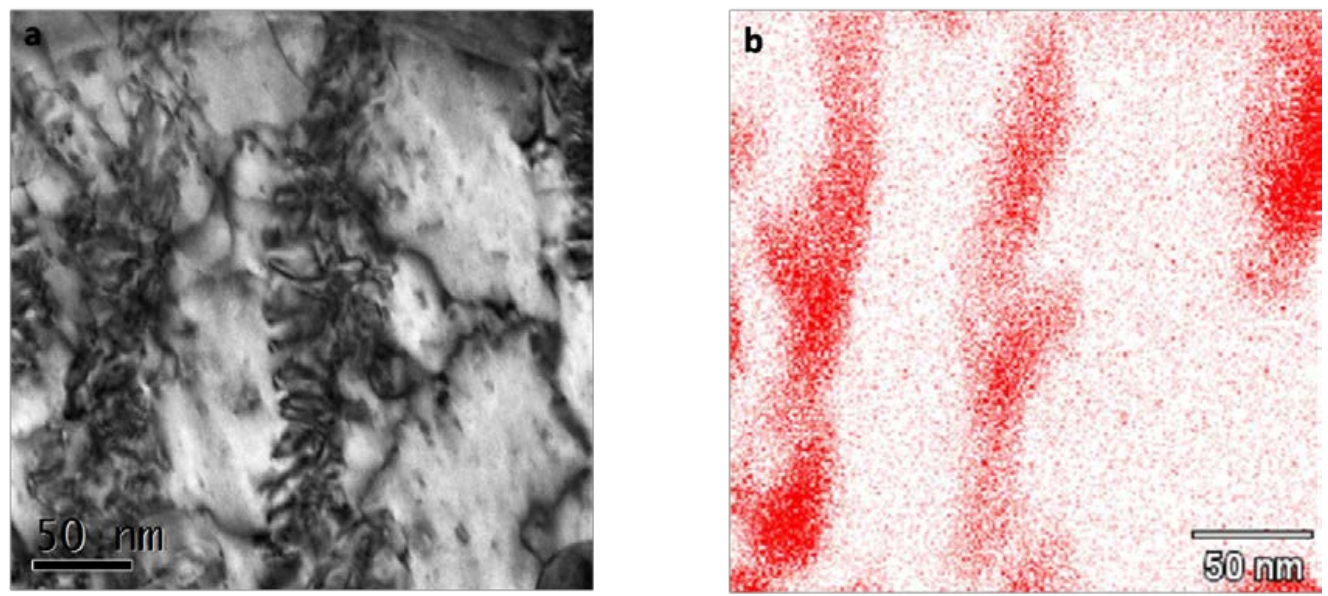

Figure 2 - (a) TEM bright field of semi-coherent DC after $60 \mathrm{~min}$ at $650^{\circ} \mathrm{C}$. (b) Corresponding EDS Co mapping.
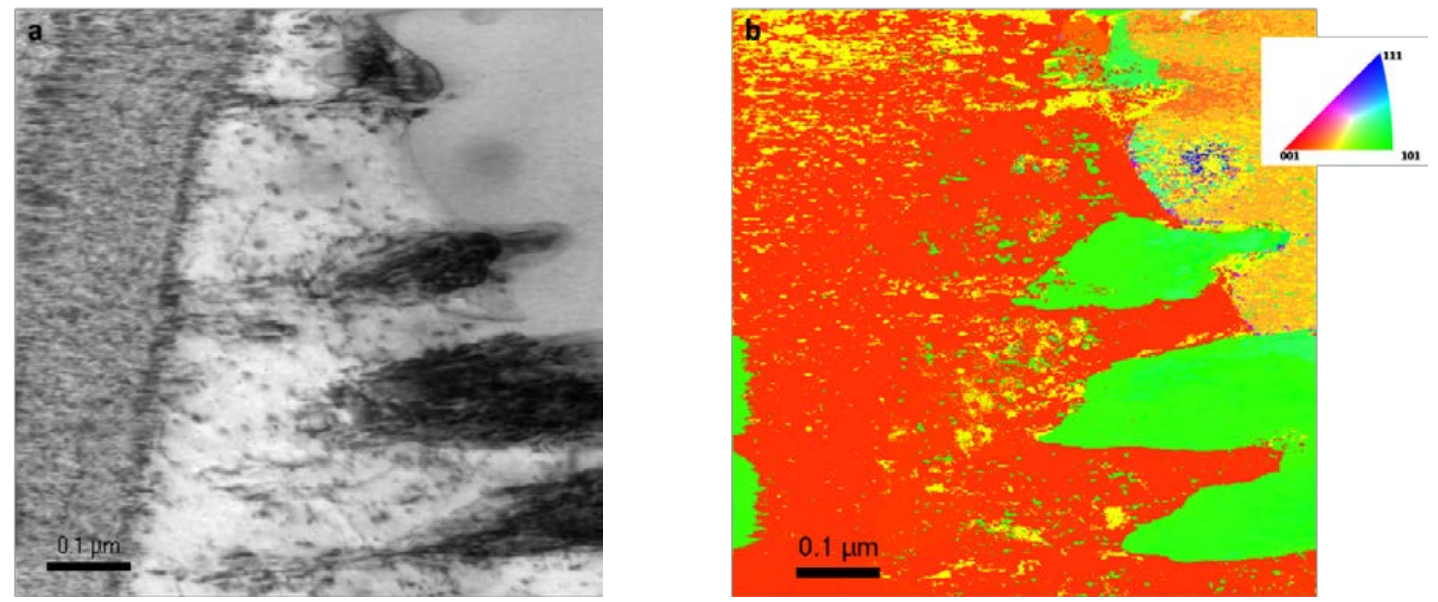

Figure 3 - NBD in a sample aged at $650^{\circ} \mathrm{C}$ for $60 \mathrm{~min}$. (a) Reconstructed bright field image. Notice the DC colonies, with precipitates with dark contrast. (b) Crystalographic orientation map. 